\title{
A Mouse Model of Chronic Pulmonary Infection with Pseudomonas aeruginosa and Pseudomonas cepacia
}

\author{
JEFFREY R. STARKE, MORVEN S. EDWARDS, CLAIRE LANGSTON, AND CAROL J. BAKER \\ Section of Infectious Diseases, Departments of Pediatrics, Microbiology \& Immunology, and Pathology, Baylor \\ College of Medicine, Houston, Texas 77030
}

\begin{abstract}
A mouse model of chronic pulmonary infection with either Pseudomonas aeruginosa or Pseudomonas cepacia was developed to compare bacteriologic and pathologic features of these infections. Experimental pneumonia was established in Swiss mice by transoral intratracheal inoculation of $10^{3}-10^{4}$ colony-forming units of mucoid $P$. aeruginosa or $P$. cepacia enmeshed in agarose beads. Unilateral infection with either strain was tolerated without morbidity. By 10 days postinoculation, the mean colonyforming units per infected lung was $3.8 \times 10^{5}$ for $P$. aeruginosa and $1.0 \times 10^{5}$ for $P$. cepacia. Bacterial counts remained stable through 21 days with no significant difference between organisms. Acute and chronic inflammatory histopathologic changes similar to many found in the lungs of cystic fibrosis patients were present in $95 \%$ of lung specimens. The changes occurred with both organisms but were more extensive with mucoid $P$. aeruginosa. This model represents an important tool for study of the contribution of complement, antibody, and adoptive transfer of $T$ cell-mediated immunity to the pathogenesis of chronic pneumonia with Pseudomonas species, and represents the first successful model of chronic pulmonary infection with P. cepacia. (Pediatr Res 22: 698-702, 1987)
\end{abstract}

\section{Abbreviations}

PA, Pseudomonas aeruginosa

PC, Pseudomonas cepacia

CFU, colony-forming units

$\mathrm{CF}$, cystic fibrosis

PBS, phosphate-buffered saline

SDC, sodium desoxycholate

LPS, lipopolysaccharide

Chronic pulmonary infection with Pseudomonas is a major factor contributing to the morbidity and mortality of at least $75 \%$ of patients with CF (1). For two decades, PA, frequently expressing a mucoid surface exopolysaccharide, has been the major species colonizing the respiratory tract of these patients $(2,3)$. Recently, some CF centers have reported an increasing number of infections with PC which may occur in association with deteriorating pulmonary function and death (4-6). Little is known about the biologic behavior and pathologic determinants of PC in human or experimental animal infections.

Direct assessment of Pseudomonas lung infections in CF pa-

Received May 11, 1987; accepted August 4, 1987.

Correspondence and reprint requests to Jeffrey R. Starke, M.D., Department of Pediatrics, Baylor College of Medicine, One Baylor Plaza. Houston. TX 77030.

J.R.S. is a Fellow of the Parker B. Francis Foundation. tients has been limited by the fact that invasive procedures such as bronchoscopy are needed to obtain lung lavage specimens. Since no animal model of CF exists, experimental models of Pseudomonas lung infection have been developed to investigate host-microbial interactions. In mice, long-term pulmonary infections previously have not been achieved in the absence of immunosuppression. In normal animals, initiation of infection by aerosolization or tracheal inoculation of organisms in saline produces transient pulmonary colonization accompanied by mild inflammatory reaction, or acute pneumonia which is fatal within 24 to $48 \mathrm{~h}$ (7-10). Cash et al. (11) developed a model of chronic PA lung infection in rats using bacteria-impregnated agar beads to initiate infection. In this model, there is proliferation of organisms and histologic damage similar to that observed in the lungs of CF patients. This model has been adapted to larger animals such as guinea pigs (12) and cats (13), but not to smaller animals. The availability of genetically defined strains as well as specific immunochemical reagents makes the mouse a theoretically ideal host for the study of immune response to chronic pulmonary infection.

The experiments reported herein fulfill two major goals. First, the technique of the agar bead method has been adapted to establish a mouse model of chronic pulmonary infection with Pseudomonas. Second, this model was employed to compare the bacteriology and histopathology of experimental PA and PC infections.

\section{MATERIALS AND METHODS}

Bacterial strains. PA strain 2192 (14) was a gift from Gerald B. Pier, Ph.D., (Channing Laboratory, Harvard Medical School, Boston, MA). This strain has a markedly mucoid appearance when grown on blood agar. The strain of PC S-1 was isolated originally at Texas Children's Hospital, Houston, TX from the sputum of an adolescent male in stable clinical condition with CF. In addition to these two strains, a nonmucoid revertant of PA 2192 (from Dr. Pier), PAO-1 (a gift from Barbara H. Iglewski, Ph.D., University of Rochester, Rochester, NY), and two additional PC isolates from patients with $\mathrm{CF}$ were employed in some experiments. Strains were stored at $-80^{\circ} \mathrm{C}$ in trypticase soy broth supplemented with glycerol.

Experimental animals. Three groups of 12 to 15 Swiss CD-1 outbred female mice, 6 to $10 \mathrm{wk}$ old and free of antibody to pathogenic murine viruses (Charles River Laboratories, Wilmington, MA) were used for each experiment. These animals were separated from other animals and were housed in the laboratory with standard care and feeding conditions.

Experimental model of infection. A suspension of bacteriaimpregnated agarose beads was made using slight modifications of previously described methods $(11,15)$. The bacteria strain to be used was grown for $18 \mathrm{~h}$ to late log phase in tryptic soy broth 
(Baltimore Biologic I aboratories Microbiology Systems. Cockcysville, MD). ^ $25-\mathrm{ml}$ aliquot of a mixture containing $0.1 \mathrm{ml}$ of the tryptic soy broth culture and $50 \mathrm{ml}$ of $2 \%$ agarose $(w / \mathrm{r})$ in PBS, pH 7.2. was pipetted forcefully into $150 \mathrm{ml}$ of heary mineral oil at $50^{\circ} \mathrm{C}$ as the oil was stirred rapidly with a magnetic stirring bar. Stirring was continued at $24^{\circ} \mathrm{C}$ for $6 \mathrm{~min}$ followed by cooling of the mixture over $10 \mathrm{~min}$. The bacteria-containing agarose beads formed were $10(0-20) \mu$ in diameter. The oilagarose mixture then was washed with $0.5 \% \mathrm{SDC}$ in PBS. The oil and SDC layers were removed by aspiration, and the washing was repeated using $0.25 \%$ SDC in PBS. followed by three washes with PBS. The washed beads were resuspended in PBS to $2-4 \times$ $10^{4} \mathrm{CHU} / \mathrm{ml}$. Actual bacterial counts were confirmed by serial dilution on blood agar plates of a hand homogenized suspension.

Mice were anesthetized lightly with ketamine hydrochloride (30) $\mathrm{mg} / \mathrm{kg}$ ) given intraperitoneally. The trachea was directly visualized by ventral midline, cervical incision. After orotracheal intubation with a 22-gauge bead-tipped stainless steel needle (Perfektum Company, New York. NY). $0.05 \mathrm{ml}$ of the bead suspension was inoculated into either both lungs or the right lung. Needles were bent at a $30^{\circ}$ angle to allow selective intubation of the right lung, and preliminary experiments employing methylene blue dye or agarose beads validated selective intubation of the right lung in more than $90 \%$ of animals. After inoculation, the incision was closed with suture. No animals developed wound infections and healing occurred in 2 to 3 days.

Bacteriologl and histopathologl: Animals were anesthetized with ether and sacrificed by retroorbital and cardiac exsanguination at 10 or 21 days after inoculation. The lungs and lower trachea were excised aseptically and selected randomly either for bacteriologic or histopathologic study. l.ungs for bacteriology were homogenized by a Polytron Homogenizer (Brinkman Instruments, Inc., Westbury, NY) and homogenates were cultured quantitatively by serial dilution on blood agar plates. $P \wedge$ and $P C$ were identified by Gram stain, colony morphology, oxidase reaction, and the rapid-NY' (DMS Laboratories. Flemington. NJ). Gram-positive organisms were identified by Gram stain, colony morphology, and catalase tests. Gram-negative fermentative bacilli were identified using the Rapid-E test (DMS Laboratories). Lungs for histopathologic study were removed en bloc and the trachea cannulated with a 20-gauge needle. They were fixed in $10 \%$ buffered formalin using an inflation-fixation apparatus constructed for the project and designed to deliver 15 to $20 \mathrm{~cm}$ of continuous intrapulmonary pressure. After $24 \mathrm{~h}$ of fixation, the lungs were sectioned and stained with hematoxylin and eosin. Coded sections were assessed blindly for extent of inflammation, bronchial epithelial alterations, alveolar changes, and fibrosis.

Statistical analysis. Because normal distributions for the measurements could not be assumed, nonparametric tests were used for statistical analysis of the data. $\wedge$ two-tailed Mann-Whitney $U$ test was used to compare colony counts between infections with PA 2192 mucoid and PC S-1 at both 10 and 21 days. The $\chi^{2}$ test with Yates correction was used to compare deaths in animals infected with $\mathrm{PA}$ and $\mathrm{PC}$.

\section{RESULTS}

Animal survival. The inoculation procedure was tolerated well by most animals. Occasionally, a mouse developed asphyxiation from the thick bead suspension, and these animals were excluded from analysis. All other animals were mobile and alert within minutes after the procedure.

Although cultures of other organs were not obtained. no animals developed clinical or macroscopic evidence of infections or other abnormalities in extrapulmonary sites. Retroorbital blood cultures obtained in selected animals at $30 \mathrm{~min}$ and 4,8 . $24,48,72$, and $96 \mathrm{~h}$ after pulmonary inoculation were consistently negative for PA 2192 mucoid and PC S-1.

$\Lambda$ number of animals inoculated bilaterally with either $\mathrm{PA}$ or
$P C$ died spontaneously before the end of the intended period of observation. Animals with bilateral pneumonia were observed only for survival: no culture or histologie studies were performed prior to death. Death from bilateral infection was significantly more common in animals inoculated with PA compared with PC (4.3 rersus 15\%, $\left.\chi^{2}=16.4 . p<0.001\right)$. All spontancous deaths occurred 4 to 9 dass after pulmonary challenge. These animals became symptomatic 24 to $48 \mathrm{~h}$ prior to death. All animals that died spontaneously had severe. bilateral pneumonia which affected all lobes of both lungs. Cultures of lung homogenates from animals selected because death was observed yielded $10^{\prime \prime}-10^{\prime \prime}$ CFU/lungs of the challenge organism. Since most of these deaths were not observed. meaningful histopathologic study of these animal's lungs could not be performed. No animal with unilateral lung infection died before experimental sacrifice. The animals with bilateral pneumonia were excluded from further bacteriologic and histopathologic analysis.

Bacteriology: The lung homogenates generally vielded the challenge organism in pure culture. Isolated colonies of staphyococei. $\alpha$-hemolytic streptococei or Proteus species were recovered occasionally. The biochemical profiles of the strains of PA and PC recovered from lung homogenates were identical to those of the inoculating strains. In no case did mucoid $P \wedge$ revert to a nonmucoid morphology.

The mean challenge doses for PA 2192 mucoid and PA S-1. and the mean CFU per lung homogenate are shown in Table 1. For each organism. the mean bacterial count increased almost two logs from inoculation to day 10 , and then remained relatively stable through day 21. The degree of bacterial proliferation was similar for PA and $P C$ at either time interval (10) days: $U=1.22$. $p=0.22: 21$ days: $U=1.61 . p=0.11)$. Rarely, animals infected with either organism had sterile lung homogenates despite the presence of obvious lobar pneumonia by gross inspection.

Pathologl: Macroscopic changes were apparent in $95 \%$ of the animals infected with either organism. and ranged from hyperemia to frank consolidation of lung tissue. Areas of consolidation were usually unilateral and affected one or two of the right lobes. The consolidated areas were dark red-brown. with a smooth but firm surface and were difficult to inflate with formalin. Fibrinous pleural adhesions frequently were present. There were no qualitative differences in gross lung appearance between $P A 2192$ mucoid and PC S-1-infected animals, or between animals sacrificed at 10 and 21 days.

Preliminary experiments showed that instillation of sterite agat beads into the mouse lung caused no local inflammatory reaction (Fig. 1). In contrast, virtually all animals inoculated with bacteriaimpregnated agar beads demonstrated both bronchial and parenchymal changes microscopically. Frequently. agar beads containing microcolonies of organisms, which were surrounded by inflammatory cells. could be seen within large or small airways (Fig. 2). The ratio of neutrophils to macrophages in this airway exudate was 8-10:1. These cells did not contain any phagocytosed bacteria. They were particularly dense near the microcotonies and in some cases appeared to invade through the surface of the agar bead (Fig. 3). There were no differences in intlammatory cell numbers or ratios between PA mucoid 2192 and PC S-1-infected lungs. Some lung sections revealed bronchial epithelial hyperplasia, while others had focal epithelial ulcerations and wide-spread squamous metaplasia. Bronchiectasis was a common finding. Proliferation of bronchus-associated lymphoid tissue occurred frequently in the peribronchial area adjacent to bead-containing airwavs. and consisted almost entirely of small lymphocytes. Assessment of a limited number of animals at 4 wh after inoculation revealed similar histopathologic findings.

A wide variety of parenchymal changes occurred, including alveolar infiltrates and interstitial inflammation. Frequently a dense necrotizing and granulomatous reaction occurred with loss of normal architecture. Early fibrosis was seen in 10-day lung sections but was more common at 21 days. White individual bacteria and bacterial microcolonies could be visualized readily 
Table 1. Bacteriologic parameters during chronic pneumonia in mice

\begin{tabular}{lcccc}
\hline Organism/strain & $\begin{array}{c}\text { Length of infection } \\
\text { (days) }\end{array}$ & $\begin{array}{c}\text { No. of } \\
\text { animals* }\end{array}$ & $\begin{array}{c}\text { Challenge dose } \dagger \\
\left(\text { CFU } \times 10^{3}\right)\end{array}$ & $\begin{array}{c}\text { Bacterial persistence } \begin{array}{c}\left(\text { CFU } \times 10^{5}\right) \\
(10\end{array} \\
\text { PA/2192 mucoid }\end{array}$ \\
PC/S-1 & 10 & 42 & $4.6 \pm 1.1$ & $3.8 \pm 1.9$ \\
PA/2192 mucoid & 21 & 45 & $5.4 \pm 1.8$ & $1.0 \pm 0.4$ \\
PC/S-1 & 21 & 43 & $4.4 \pm 1.3$ & $2.3 \pm 1.6$ \\
\hline
\end{tabular}

* Sum of animals from three separate experimental groups.

$\dagger$ Geometric mean $( \pm \mathrm{SE}$ ) of CFU/0.05 $\mathrm{ml}$ of inoculum suspension of beads for three groups of animals.

$\ddagger$ Geometric mean $( \pm S E)$ of CFU in lung homogenate per animal.

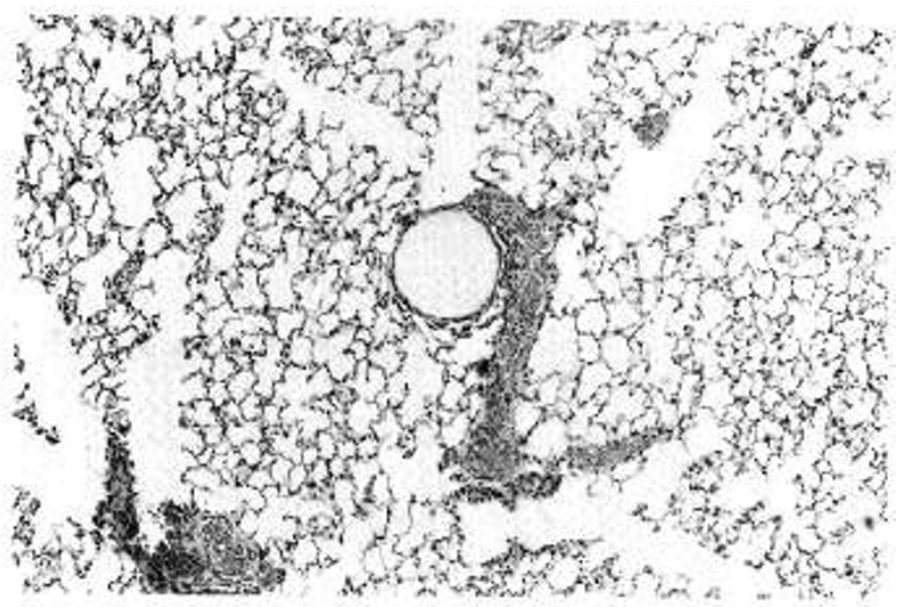

Fig. 1. Lung of an animal with sterile beads for 10 days. There is no surrounding inflammatory reaction (hematoxylin and cosin, $\times 150$ ).

within the beads, no free bacteria were seen by light microscopy within the airways or the parenchyma. In general, the blood vessels had normal architecture and were not inflamed.

The appearance of the agar beads, airway inflammation, numbers and types of inflammatory cells, and parenchymal infiltrates caused by PA 2192 mucoid and PC S- 1 were indistinguishable. However, the parenchymal damage caused by PA 2192 mucoid was $50 \%$ more extensive by area, spreading more distally from bead-containing airways. Results with PA 2192 nonmucoid revertant and PAO-1 were identical to those with PA 2192 mucoid, and those with our other two PC strains were indistinguishable from PC S-1.

\section{DISCUSSION}

The agar bead model of chronic pneumonia has been adapted successfully to several animal species $(12,13)$. Previous studies using this model have examined the ability of certain Pseudomonas products to either promote pulmonary tissue damage or to be used as vaccines. Except for measurements of antibody titers to certain bacterial products, studies of the host's immunologic responses to chronic pulmonary infection are lacking. Several characteristics of the mouse make it a unique host for immunologic studies. First, the genetic diversity of mouse strains will enhance the study of the role of specific bacterial products and components of the host response in pulmonary pathogenesis. For instance, the contributions of LPS and surface polysaccharides to tissue damage and animal protection can be examined in mouse strains with genetically determined exaggerated or diminished response to these antigens. Similarly, the importance of complement in bacterial clearance and immune complex formation can be determined using normal animals and congenitally complement-deficient mutants. Second, a much wider array of immunochemical reagents are available for the mouse than other animals, augmenting the ability to determine the

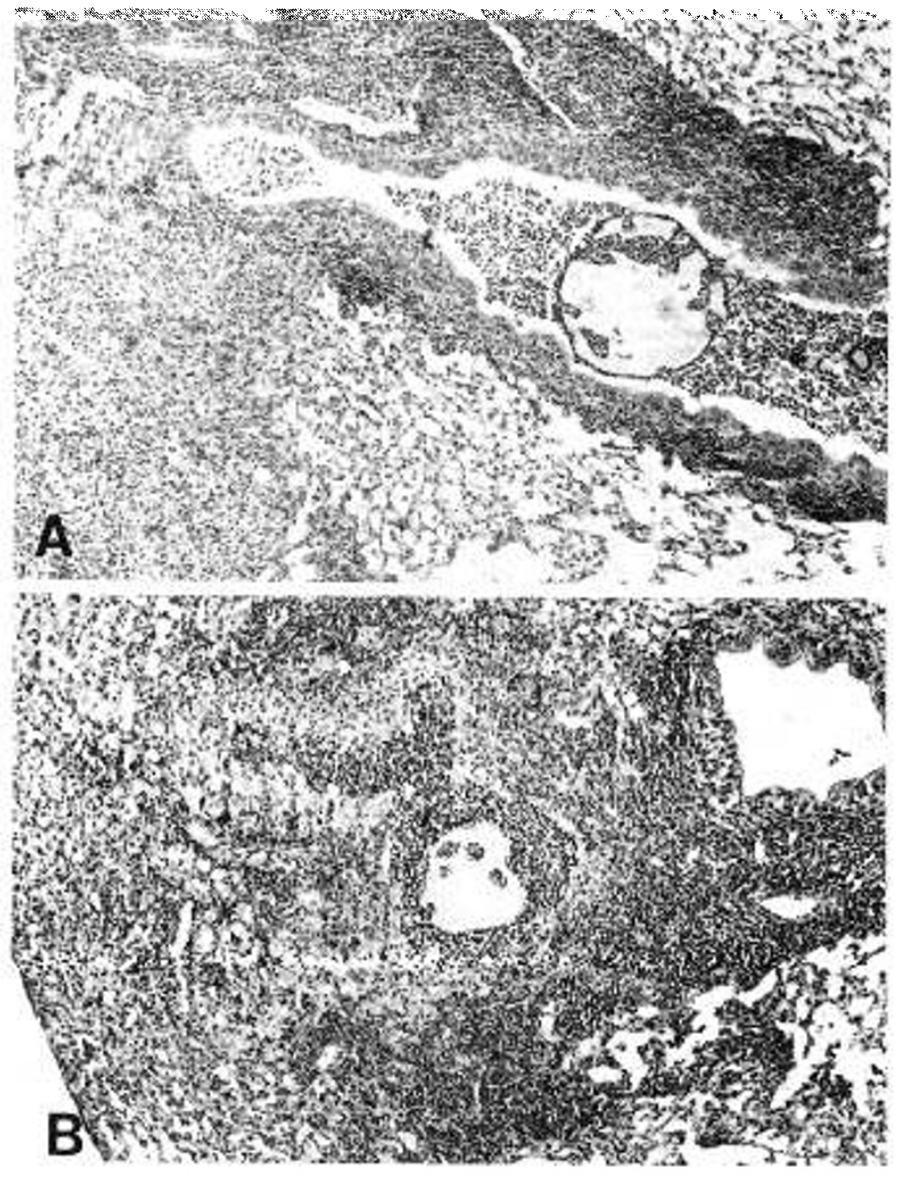

Fig. 2. Lung from animals infected for 10 days with agar beads containing PA 2192 mucoid $(A)$ and PC S-1 $(B)$. The airways are filled with an exudate consisting predominately of neutrophils with some macrophages. The surrounding parenchyma is obscured by a mononuclear cell inflammatory response. Focal destruction and squamous metaplasia of the bronchiolar epithelium can be seen $(A)$. The airway epithelium is ulcerated widely $(B)$. The degree of inflammation seen $(B)$ is unusually severe for infection with PC S-1 (hematoxylin and eosin, $x$ 150).

importance of specific antibody subclasses and leukocyte cell surface components in host pulmonary defense. Finally, since previous studies have demonstrated an important contribution of $\mathrm{T}$ cell-mediated immunity against Pseudomonas surface polysaccharide in mice (16), studies of protection against lung infection using adoptive transfer of leukocytes could be performed in a mouse model of infection.

Before experiments suggested by these characteristics can be performed, a mouse model of chronic lung infection must be defined. Previous attempts using aerosolization (10) or direct tracheal instillation of organisms in saline $(7,9)$ have produced, 


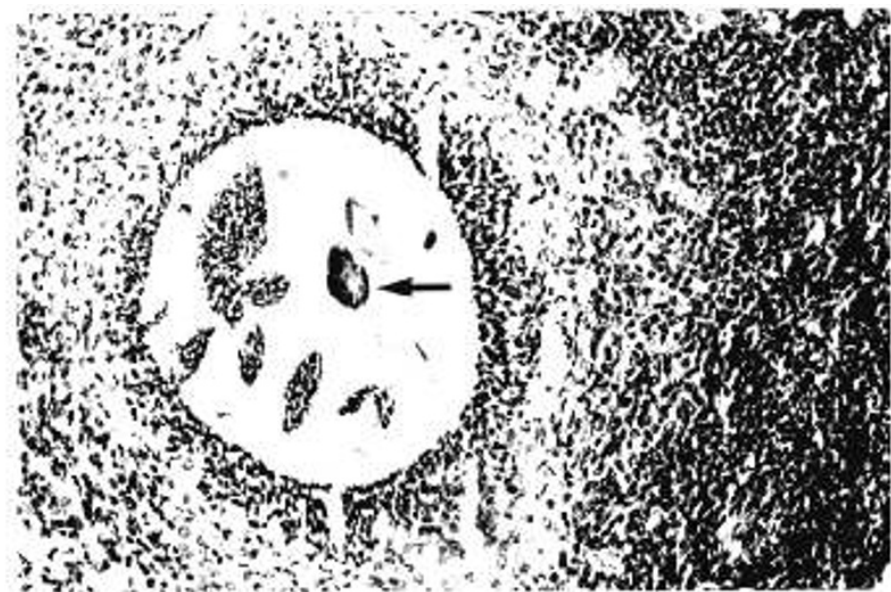

Fig. 3. An agar bead containing. P( $S-1$ in a mouse infected for 21 days. Several microcolonics (armen) can be secon, some of which are covered with neutrophils. At higher power. individual organisms ane seen casily within these microcolonies. The bronchial epithelium is complecely destroyed (hematoxylin and cosin, $\times 3(0)$ ).

depending on the inoculum, cither transient, mild inflammatery reactions with complete bacterial clearance. or acute fatal pneumonia. The present experiments have demonstrated that mice subjected to unilateral transtracheal inoculation with Pseudomonas-impregnated agat beads coukd maintain an inflammators response for at least $3 \mathrm{wk}$. The results were similar to those observed in larger animals (11, 12). Bacterial counts increased in the days following inoculation by $10(0)$ fold. and then remained stable throughout the remaining course of infection. The histopathologic lesions seen in mice were similar to some lound at autopsy in ( I: patients with chronic pulmonary discase (17). Both naturally occurring and cxperimental infections frequently manifest bronchopneumonia. bronchiectasis, mucus plagging. epithelial metaplasia. fibrosis and alveolar exudates with acute and chronic inflammatery cells. I ymphoid hyperplasia. which was prominent in the infected animals. also is noted frecterents in the lungs of ( $I$ patients and is believed to be a consequence of chronic antigenic stimulation (18). Pulmonary vascular inflammatory lesions. which are common among paticnts with acute bacteremic Pseudomonas pneumonia. were not observed in ( $F$ patients or in chronically infected animals.

Although PA has been the predominant pathogen colonizing the lungs of ( $I$ patients during the last $1 w 0$ decades. P( recenty has emerged as a serious cause of morbidity and mortality. Some

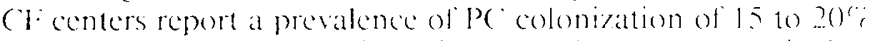
(5.6). Pulmonary acquisition of P' 'mav be asvmptomatic. but it is often followed by a gradual or very rapid decline in clinical status. Sistemic signs of infection and bacteremia. Which are rarely associated with $P \wedge$ infection in (I patients. oceur commonly in patients colonised with PC (6). The pathogenesis of $P($ in ( $I$ patients is unknown, and the host immune response to PC has not been investigated.

No previous studies of experimental Pe pneumonia have been reported. P' pneumonia is not easily established in the guinea pig and chronic infection in the rat has been described only superficially (19). In our model the findings with P( were similar to those with $P \wedge$. The increase in hacterial (I:I was the same for the wo organisms. and both appeared to grow in micrexolonies within the agal beads. I ssing electron microscop)y. I am et at. (20) showed that PA obtained from bronchial mucus of ( $\mathrm{F}$ patients and from rats with agar-bead-indeced chronic pneumonia formed microcolonies that were enveloped in a fibrous anionic matrix. These investigators postulated that this form of growth protected the organism from bost defense mechanisms. but also limited its toxicity and dissemination (21). Although P( growth has not been studied similarly. We were able to demonstrate microcolons growth within agal beads by light microseops.

The pathologic processes associated with PA and PC were qualitatively the same. although the were stightly morecxtensive in the $P A$ groups of animals. The increased lethality of hilateral pneumonia with PA compared to $P\left(C^{\prime}\right.$ is consistent with the more extensive disease also seen in unilateral intection. The differences observed between $P \wedge 2192$ and P' ' $S-1$ mat be a reflection of strain rather than species chatracteristics. In smaller groups of mice, the results with 1 wo additional strains of P: 1 were indistinguishable from those of mucoid P : 2192. whik two other strains of P' obtained from ("F patients vidded results identical to those with PC S-1. For more meaningtul comparisons to be made. future studies employing more well-detined strains of $P A$ and P( will have 10 be undertaken. In this wats, the role of specilie virulence factors such as rough rewas smooth l.PS. mucoid polysaccharide. and specitic proteases and tosins. could be defined.

One disadrantage of the agar-head infectom modet is that it does not simulate the natural history of Pseudomonas infection in ( $\mathrm{F}$ : from arrwaty coloniattion to lung injurs. However. the type of tissue damage and the host response to both P $P$ and $P C$

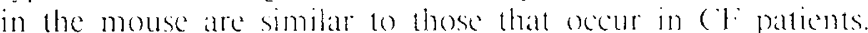
Therefore. this model can be used with normal animals as well as those with diminished response's to l.PS in the cualuation of the contributions of complement. antibodies of various clatses and subclasses. cell-mediated immunc function. and immunc complex deposition to protection from or promotion of tissue damage in chronic Pseudomonas pulmonary infection.

Whomledement. The authors thank Rohin Dudlew for help with preparation of this manuscript.

\section{RETIRKIVIR}

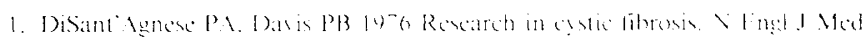

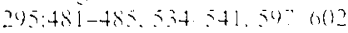

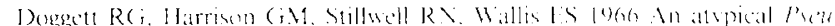

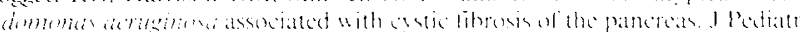
$0,8: 215-2.210$

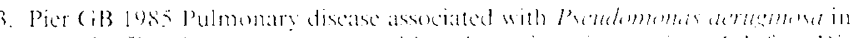

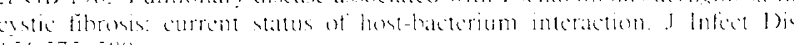
151.5755580

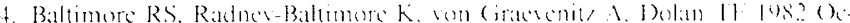

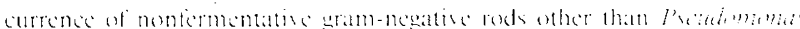

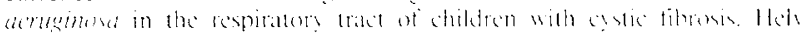
Pacdiat leta $37: 547 ? 54$

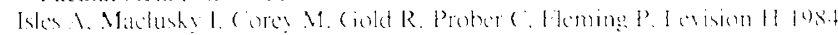

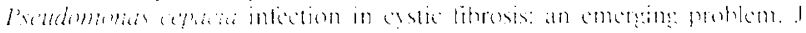

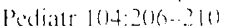

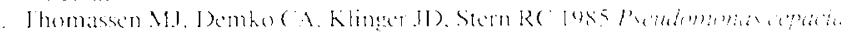

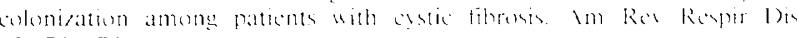
$1,31: 791706$

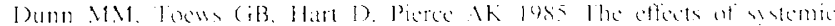

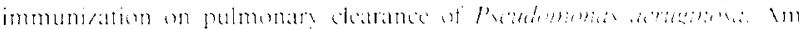
Rer Respir Dis $131+426+4 i$

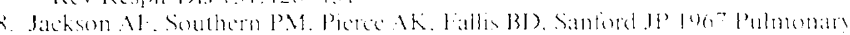

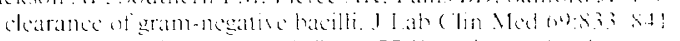

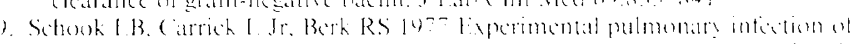

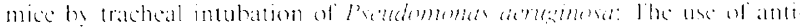

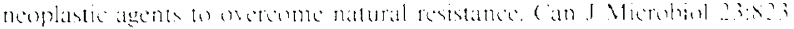
$\ll 26$

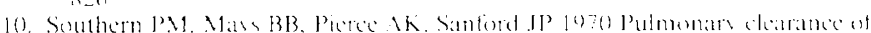

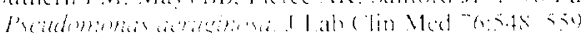

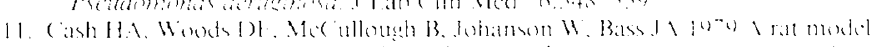

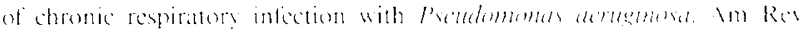

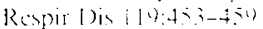

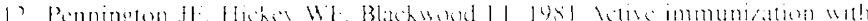

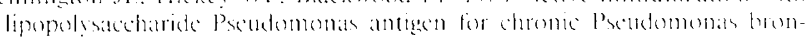

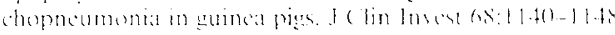

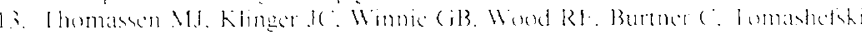

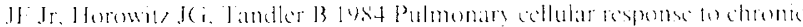

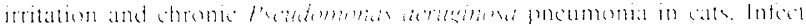
Immun $4=0+10$

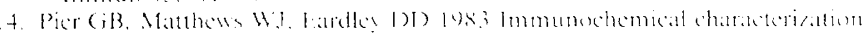

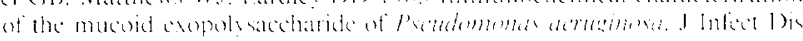


147:494-503

15. Klinger JD. Cash HA, Wood RE, Miler JJ 1983 Protective immunization against chronic Pscudomonas acruginosa pulmonary infection in rats. Infect Immun 39:1377-1384

16. Pier GB, Markham RB 1982 Induction in mice of cell-mediated immunity to Pseudomonas aeruginosa by high molecular weight polysaccharide and vinblastine. J Immunol 128:2121-2125

17. Bedrossian CWM. Greenberg SD. Singer DB. Hansen JJ. Rosenberg HS 1986 The lung in cystic fibrosis. A quantitative study including prevalence of pathologic findings among different age groups. Hum Pathol 7:195-204
18. Esterly JR. Oppenheimer EH 1968 Observations in cystic fibrosis of the pancreas. III. Pulmonary lesions. Johns Hopkins Med J 122:94-101

19. Goldman DA, Klinger JD 1986 Pseudomonas cepacia: biology mechanisms of virulence, epidemiology. J Pediatr 108:806-812

20. Lam J. Chan R. Lam K. Costerton JW 1980 Production of mucoid microcolonies by Pseudomonas aeruginosa within infected lungs in cystic fibrosis. Infect Immun 28:546-556

21. Costerton JW, Lam J, Lam K, Chan R 1983 The role of the microcolony mode of growth in the pathogenesis of Pseudomonas aeruginosa infections. Rev Infect Dis 5:S867-S873

\section{Announcement}

\section{FASEB Summer Research Conferences 1987 Series Completed, 1989 Topic Proposals Sought}

The sixth summer of the FASEB Summer Research Conference series has been completed. This summer there were 17 conferences held at two conference sites: Saxtons River, Vermont, and Copper Mountain, Colorado. The series was established to complement the large FASEB Annual Meeting. It was designed to provide quiet settings to encourage a relaxed exchange of information on the cutting edge of biomedical research. More than 1900 scientists from the United States and 18 foreign countries enjoyed the exciting science that was presented and discussed at the meetings in 1987.

Many of the 1987 conference participants requested that their conference topics be rescheduled in the future FASEB Summer Research Conference series. Topic proposals are required from potential organizers of these conferences, as well as from those proposing new topics for consideration by the Conference Advisory Committee at its fall meeting

The committee is seeking new topic proposals on subjects of keen interest to biological and medical researchers for fields that require concentrated 5-day programs for adequate discussions on the latest developments. A topic proposal outline is available from Dr. Robert W. Krauss, FASEB Executive Office (301-530-7093) to assist in the submission of proposals for FASEB Summer Research Conferences in 1989 or 1990.

The topics for the 1988 series have been set and are outlined below. The Conference Advisory Committee will meet in October or November of this vear to establish the 1989 schedule and a tentative schedule for 1990.

\section{FASEB Summer Research Conferences}

\section{Saxtons River, V'T}

Yeast RNA: Transcription, Splicing, Translation, Replication and Transposition June 12-17

Chairperson: Reed B. Wickner, NIADDK. NIH

Retinoids June 19-24

Chairperson: DeWitt S. Goodman. Columbia University College of P\&S

Smooth Muscle June 26-July 1

Chairperson: R. Kent Hermsmeyer, University of Nevada School of Medicine

Autoimmunity July 3-8

Chairperson: Howard L. Weiner. Brigham and Women's Hospital

Phospholipases July 10-15

Chairperson: Moseley Waite. Bowman Gray School of Medicine

Immunopharmacology July 17-22

Chairperson: Timothy Sullivan, University of Texas Health Science Center-Dallas

Structure and Function of Cell Membranes July 24-29 Chairperson: Philip L. Yeagle, SUNY at Buffalo

Somatic Cell Genetics July 31-August 5 Chairperson: Geoffrey Wahl, The Salk Institute

Receptors August 7-12

Chairperson: Richard Klausner, National Institutes of Health

Electrophysiological Mechanisms of Propagation in and Activation of Cardiac and Smooth Muscle August 14-19 Chairperson: David R. Harder, Medical College of Wisconsin
Copper Mountain, $\mathrm{CO}$

Neuroimmunomodulation July 26-July 1 Chairperson: Novera H. Spector, University of Alabama at Birmingham

Ultradian and Infradian Modulation of the Circadian System July 3-8

Chairperson: Lawrence E. Scheving, University of Arkansas

Regulation of Gene Expression in Higher Animals by Hormones and Nutritional Substrates July 10-15

Chairperson: George A. Scheele, The Rockefeller University

Molecular Biology of Infectious and Parasitic Diseases July $17-22$

Chairperson: Richard A. Young, Whitehead Institute for Biomedical Research

Trichothecene, Blue-Green Algal and Marine-Toxins: Mechanisms, Detection and Therapy July 24-29

Chairperson: Adrianne Rogers, Boston University School of Medicine

Folic Acid, Vitamin B-12 and One-Carbon Metabolism July 31-Aug 5

Chairperson: Raymond Blakely, St. Judes Children's Research Hospital

Endothelium and Cardiovascular Function August 7-12 Chairperson: Paul M. Vanhoutte, Mayo Foundation

Neoplastic Transformation of Liver Cells August 14-19 Chairperson: Snorri Thorgiersson, National Cancer Institute, $\mathrm{NIH}$ 